\title{
Quantification of Compositional and Residual Stress Effects on Lattice Strain in Dual-phase Stainless Steels by Means of Differential Aperture X-ray Micro-diffraction
}

\section{Nan $L I^{1)}$, Zhinan $A N^{2}$, Wenjun $L I U^{3)}$ and Yandong $W A N G^{4) \dagger}$}

1) School of Materials Science and Engineering, Beijing Institute of Technology, Beijing 100081, China

2) Department of Materials Engineering, the University of Tennessee, Knoxville, TN 37996, USA

3) Advanced Photon Source, Argonne National Laboratory, Argonne, IL 60439, USA

4) State Key Laboratory of Advanced Metals and Materials, University of Science and Technology Beijing, Beijing 100081, China

[Manuscript received 31 May 2013, in revised form 24 June 2013]

(c) The Chinese Society for Metals and Springer-Verlag Berlin Heidelberg

\begin{abstract}
Residual stress is an important factor for evaluating the deformation and failure of engineering materials. Diffraction-based measurement assumes that the full measured lattice strain tensor contributes to residual stress according to Hooke's Law. The present work focuses on the lattice strain determination of individual grains in a dual-phase stainless steel (DPSS) by means of differential-aperture X-ray micro-diffraction (DAXM). The results show that the residual stress only takes part of the responsibility of the total measured lattice strain. In fact, the compositional variation inside the material was found to cause greater strain gradient in both ferrite $(\alpha)$ and austenite $(\gamma)$ phases in DPSS. Therefore, quantification of compositional and residual stress effects on lattice strain was conducted in order to evaluate the true residual stress inside engineering materials.
\end{abstract}

KEY WORDS: Differential aperture X-ray mico-diffraction; Lattice strain; Residual stress; Dual-phase stainless steels

\section{Introduction}

Residual stress is the stress that remains after the original cause of the stress (external forces, heat gradient) has been removed. All residual strain $\left(\varepsilon_{h k l}\right)$ measurement based on diffraction was calculated with the use of Eq. (1) through measuring the average $d$ spacing of plane $(h k l)$ of bulk polycrystalline materials $\left(d_{h k l}\right)$ and comparing with the reference state $\left(d_{h k l}^{0}\right)^{[1]}$.

$$
\varepsilon_{h k l}=\left(d_{h k l}-d_{h k l}^{0}\right) / d_{h k l}^{0}
$$

This method takes into account of two factors that affect the change of $d$-spacing: the residual stress, and the compositional variation. Yet, previous investigations $^{[2-4]}$ on residual stresses did not consider the compositional effect. In fact, the compo-

\footnotetext{
† Corresponding author. Prof.; Tel.: +86 10 68918480; E-mail address: ydwang@mail.neu.edu.cn (Yandong WANG)
}

DOI: $10.1007 / \mathrm{s} 40195-013-0328-0$ sitional variations, such as precipitation/segregation or chemical gradient, may make huge contribution to the change of measured lattice parameters ${ }^{[5-11]}$. If the compositional effect is not considered, the stress measurement may not be reliable, which potentially leads to catastrophic structural integrity assessment in industrial applications. Therefore, the evaluation of compositional effect and residual stress effect in determination of lattice parameter change is a significant work, especially in engineering applications.

In this work, differential-aperture X-ray microscopy (DAXM), a powerful tool in mapping the lattice parameter inside individual grains unambiguously ${ }^{[12]}$, was applied to measure the lattice parameters of a dual-phase stainless steel (DPSS). In this method, white-beam DAXM measures deviatoric (shear) strains with sub-micrometer resolution in three dimensions (3D); scanning-monochromatic DAXM provides absolute measurements of dilatational lattice parameters, and hence dilatational 
Table 1 Chemical composition of the duplex stainless steel (wt.\%)

\begin{tabular}{cccccccccc}
\hline $\mathrm{Cr}$ & $\mathrm{Ni}$ & $\mathrm{Mn}$ & $\mathrm{Mo}$ & $\mathrm{Si}$ & $\mathrm{S}$ & $\mathrm{C}$ & $\mathrm{N}$ & $\mathrm{P}$ & $\mathrm{Fe}$ \\
\hline 25 & 7 & 1.2 & 4 & 0.8 & 0.02 & 0.03 & 0.3 & 0.035 & Bal. \\
\hline
\end{tabular}

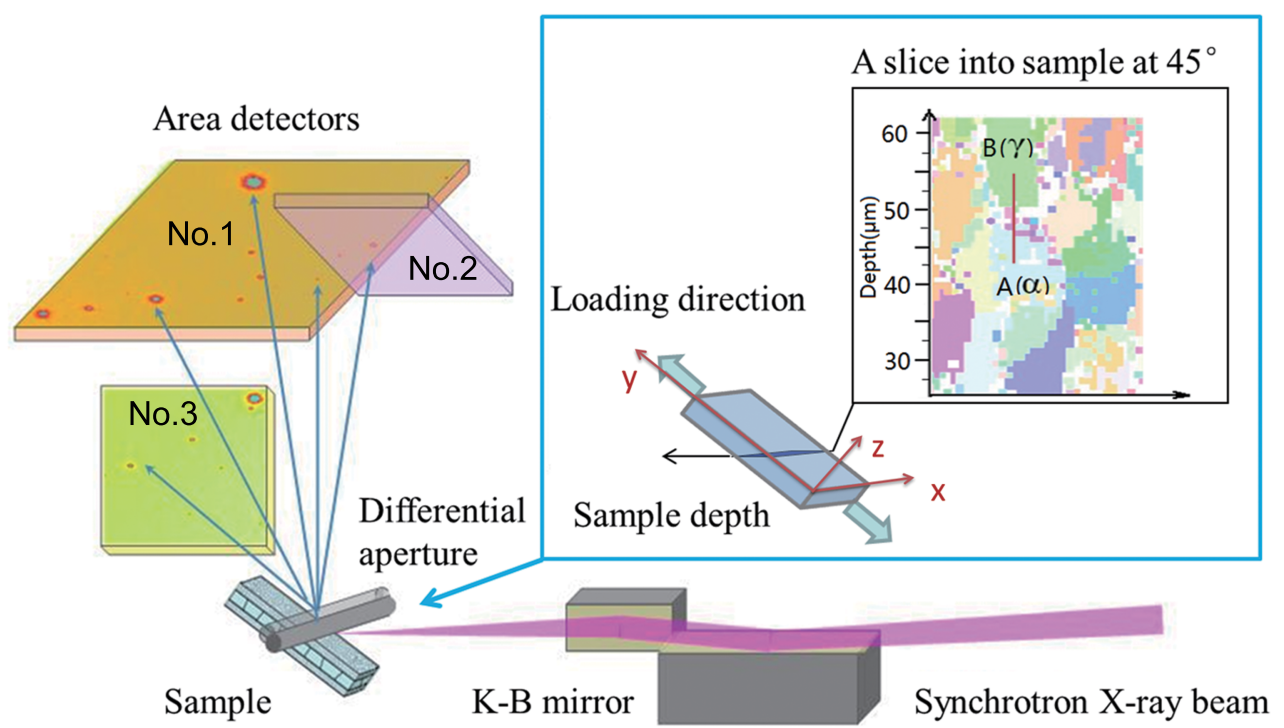

Fig. 1 The 3D-DAXM experiment set-up. A representative slice at the sample depth direction was indexed. The orientation map was inserted in the top-right of this figure. Mono-beam energy scan was conducted along the red line from grain A to grain B

strains, with 3D sub-micrometer resolution ${ }^{[13]}$.

\section{Experiment}

DPSS is used as the material studied in the present work. The austenitic-ferritic $(\gamma-\alpha)$ structures of this DPSS may have both composition segregation and phase-to-phase interaction induced internal stress; and thus is an ideal target material for the current research.

The studied DPSS is a commercial super-duplex stainless steel, SAF 2507, provided by Sandvik AB. The as-received hot-rolled bar is $20 \mathrm{~mm}$ in diameter. After solution annealing and water quench treatment, the DPSS is free of grain boundary carbides and intermetallic phases. Its nominal chemical composition is presented in Table 1.

The 3D-DAXM was carried out by means of synchrotron X-ray diffraction at beamline 34-ID-E in Advanced Photon Source (APS) at Argonne National Laboratory (Argonne, IL, US). The experimental setup is shown in Fig. 1. The synchrotron X-ray beam enters from right and hits on the sample which is mounted with an angle of $45^{\circ}$ to the horizontal plane. With the purpose of distinguishing the compositional and residual stress effects, three detectors were allocated to measure the absolute lattice parameters of the same position within the identical grain, a center $2 \mathrm{D}$ area detector positioned at $90^{\circ}$ to the incident beam and two side detectors, with large solid angles, on each side of the center one. A standard strain-free silicon single crystal was used to calibrate the three detectors' geometry with respect to the incident beam. The energy range for the current experiment was $15-30 \mathrm{keV}$, and the beam size was $0.5 \mu \mathrm{m} \times 0.5 \mu \mathrm{m}$. There are two modes of the beam: polychromatic beam (white beam), which is used to measure the local phase/crystal orientation (microtexture) and elastic/plastic deviatoric strain tensors; and monochromatic beam, which is required for absolute lattice parameter measurements.

\section{Results and Discussion}

\subsection{Depth direction grain structure mapping}

A slice scan was made along the $\mathrm{x}$-axis and the beam penetration direction. Typically, a $\mathrm{Pt}$ wire has to be stepped along the sample surface, which is $45^{\circ}$ from the incident beam, so as to obtain spatial resolved Laue patterns ${ }^{[14]}$. Then, the depth resolved diffraction patterns were indexed using computer cluster and reconstructed to a $2 \mathrm{D}$ orientation map (Fig. 1). Due to the coexistence of $\gamma$ and $\alpha$ phases in DPSS, fcc and bcc structures were both used to index the Laue patterns in order to obtain the orientation map. A typical orientation map is shown as the colored map in Fig. 1. In this figure, different colors represent different grains, among which were identified as ferrite $(\alpha)$ phase with bcc structure for grain A and 
austenitic $(\gamma)$ phase with fcc structure for grain B. Mono-beam diffraction was applied in order to check the two grains' crystal structures by measuring the accurate lattice parameter of a random position inside each grain. The lattice parameter $(a)$ was calculated with Eq. (2):

$$
a=\frac{2 \pi}{Q} \sqrt{h^{2}+k^{2}+l^{2}}
$$

where $Q$ is the modulus of the diffraction vector. As a result, the lattice parameters of grains $\mathrm{A}$ and $\mathrm{B}$ are $0.2861 \mathrm{~nm}$ and $0.3612 \mathrm{~nm}$, respectively, which exactly certified them to be $b c c$ ferrite-phase and $f c c$ austenite-phase. Taking those two grains as candidates, mono-beam energy scan was conducted to investigate the lattice strain evolution across the two grains, as indicated by the red line in Fig. 1.

\subsection{Effects of composition and residual stress on lat- tice parameter determination}

Due to crystal elasticity, residual stress will cause anisotropic lattice distortion ${ }^{[15]}$, resulting to deviatoric lattice strain. The stress-strain relationship follows Hooke's law:

$$
\sigma_{i j}=C_{i j, k l} \varepsilon_{k l}
$$

where $\sigma$ is the stress, $\varepsilon$ is the strain, and $C$ is the stiffness coefficients.

The compositional effect will generally cause an isotropic lattice expansion or shrinkage in cubic systems. At atomic level, considering an interstitial or a larger substitutional atom inside a cubic lattice, the neighboring atoms will be expelled from their original sites, resulting in an expansion of the lattice, as shown in Fig. 2(a) and (b). For a dilute system, all the neighboring atoms are the same. Since the interaction of the central and the neighboring atoms are spherical symmetric and the lattice structure is cubic, the interaction force between the central atom and its neighbors are the same. Thus, the lattice expansion can be simplified as isotropic. Similar isotropic shrinkage will also occur if the central atoms are voids or smaller substitutes. In fact, it may not always be the case that all neighboring atoms are the same; thus, the localized atomic interaction may have certain anisotropy. However, due to the random distribution of interstitial or substitutional atoms in the lattice structure, such anisotropy can be averaged if the measured volume is large enough. In the present X-ray micro-diffraction study, the beam size is about $0.5 \mu \mathrm{m} \times 0.5 \mu \mathrm{m}$, which is large enough to have the compositional effect averaged into isotropic effect. The studied material DPSS which is Fe-based alloy, with two phases being cubic structures (fcc and bcc). Therefore, it is valid to assume that the compositional effect in this material will lead to isotropic lattice strain.

The separation of the contribution from compositional variation and residual stress is determined by (a)

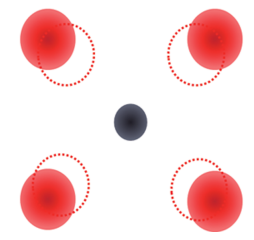

(b)

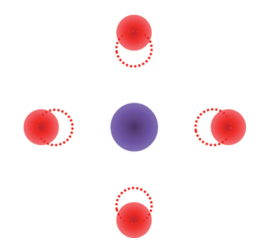

Fig. 2 Schematic atomic configuration in a cubic system with an interstitial (a) or a substitutional (b) atom. The red balls represent the neighboring atoms expelled from their original sites (dashedcircles) due to the interstitial atom (dark blue) or the substitutional atom (purple)

the component of dilatational strain and deviatoric strain. The full strain tensor of a position in a crystal contains both the hydrostatic dilatational strain and a distortion deviatoric strain term that can be expressed as:

$\varepsilon=\left(\begin{array}{lll}\varepsilon_{11}-\frac{\Delta}{3} & \varepsilon_{12} & \varepsilon_{13} \\ \varepsilon_{21} & \varepsilon_{22}-\frac{\Delta}{3} & \varepsilon_{23} \\ \varepsilon_{31} & \varepsilon_{32} & \varepsilon_{33}-\frac{\Delta}{3}\end{array}\right)+\left(\begin{array}{ccc}\frac{\Delta}{3} & 0 & 0 \\ 0 & \frac{\Delta}{3} & 0 \\ 0 & 0 & \frac{\Delta}{3}\end{array}\right)$

Due to cubic symmetry, the sum of diagonal elements of the distortion strain tensors has to be zero, hence the strain tensor can be written with five unknowns as shown in Eq. (5):

$$
\varepsilon=\left(\begin{array}{lll}
\varepsilon_{11}^{*} & \varepsilon_{12} & \varepsilon_{13} \\
\varepsilon_{12} & \varepsilon_{22}^{*} & \varepsilon_{23} \\
\varepsilon_{13} & \varepsilon_{23} & -\varepsilon_{11}^{*}-\varepsilon_{22}^{*}
\end{array}\right)+\left(\begin{array}{ccc}
\frac{\Delta}{3} & 0 & 0 \\
0 & \frac{\Delta}{3} & 0 \\
0 & 0 & \frac{\Delta}{3}
\end{array}\right)
$$

where $\varepsilon_{i i}^{*}=\varepsilon_{i i}-\Delta / 3$.

\subsection{Experimental quantification of compositional and residual stress induced lattice strain}

The Laue diffraction pattern of grain A ( $\alpha$ phase) was collected by three $2 \mathrm{D}$ area detectors and shown as in Fig. 3. The red circles represent the featured planes (356), (237) and (114) that satisfied the mono-beam Laue diffraction.

Mono-beam energy scan was applied to measure the accurate $d$-spacing of a specific $h k l$ plane ${ }^{[16]}$. With the use of three detectors, the $d$-spacing of three different $h k l$ planes can be measured simultaneously. The full strain tensor is then able to be calculated, and the contribution from the residual stress and composition could be quantitatively calculated by using Eq. (5). Taking the (356), (237) and (114) diffraction data of $\alpha$ phase as examples, the detailed calculation is described as follows:

(1) The mono-beam energy scan was conducted in a small energy range $(\Delta E)$ with 500 steps. After integrating the 500 diffraction Laue patterns, a pseudo-white-beam diffraction pattern was obtained. 
Table 2 The scatter vector $\boldsymbol{q}$ of three $h k l$ planes from the three detectors in Fig. 2

\begin{tabular}{cccc}
\hline Detector & Scatter vector $(\boldsymbol{q})$ & Modulus of $\boldsymbol{q}(Q)$ & $(h k l)$ \\
\hline No.1 & $(0.121052,0.5878427,-0.7998671)$ & 171.881 & $(237)$ \\
No.2 & $(-0.379538,0.47312,-0.79505)$ & 185.254 & $(-228)$ \\
No.3 & $(0.3461561,0.4026293,-0.8473875)$ & 182.534 & $(356)$ \\
\hline
\end{tabular}

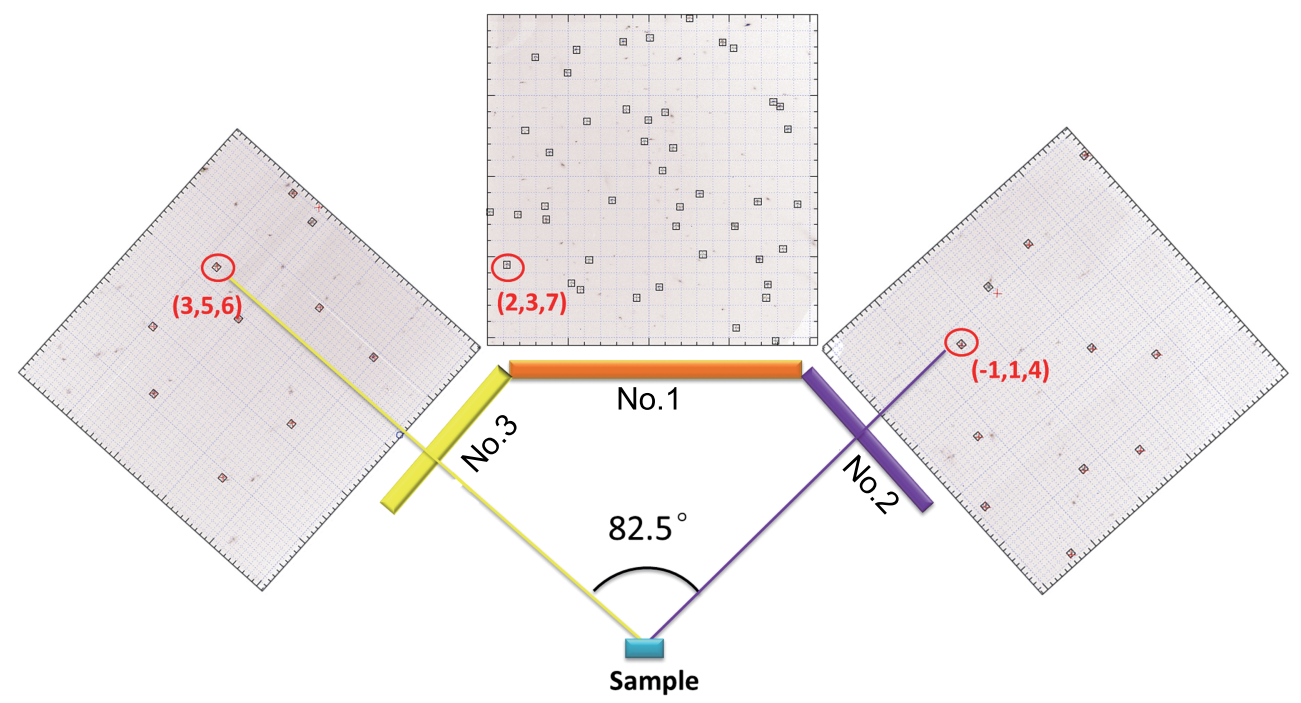

Fig. 3 The arrangement of three 2D detectors collecting Laue patterns. Three highlighted Laue diffraction spots on three detectors, (237) on No.1 detector, (114) on No.2 detector, and (356) on No.3 detector were used for the calculation of lattice strain of grain $\mathrm{A}$

(2) The pseudo-white-beam Laue diffraction pattern was analyzed by a computer cluster in the beamline 34-ID-E so as to fit the $\boldsymbol{q}$ vectors of all Laue patterns.

(3) After selecting one $\boldsymbol{q}$ vector from each detector, three $\boldsymbol{q}$ vectors from three different $h k l$ planes were obtained, as listed in Table 2.

(4) After normalizing the indexed $\boldsymbol{q}$ vector, its true value was obtained with production of the normalized $\boldsymbol{q}$ vector and the modulus, $Q$. The three $\boldsymbol{q}$ vectors were then described as:

$$
\left\{\begin{array}{l}
\boldsymbol{q}_{1}=(3.31148,16.08085,-21.88093) \\
\boldsymbol{q}_{2}=(-11.1903,13.9495,-23.44141) \\
\boldsymbol{q}_{3}=(10.0563,11.69686,-24.6176)
\end{array}\right.
$$

In reciprocal space, the $\boldsymbol{q}$ vectors can be described as:

$$
\left\{\begin{array}{l}
\boldsymbol{q}_{1}=h_{1} \boldsymbol{a}^{*}+k_{1} \boldsymbol{b}^{*}+l_{1} \boldsymbol{c}^{*} \\
\boldsymbol{q}_{2}=h_{2} \boldsymbol{a}^{*}+k_{2} \boldsymbol{b}^{*}+l_{2} \boldsymbol{c}^{*} \\
\boldsymbol{q}_{3}=h_{3} \boldsymbol{a}^{*}+k_{3} \boldsymbol{b}^{*}+l_{3} \boldsymbol{c}^{*}
\end{array}\right.
$$

where $\boldsymbol{a}^{*}, \boldsymbol{b}^{*}$ and $\boldsymbol{c}^{*}$ were the three primitive vectors in reciprocal space. Combining Eq. (6) and Eq. (7), the reciprocal primitive vectors were then obtained as:

$$
\left\{\begin{array}{l}
\boldsymbol{a}^{*}=(0.251740,0.131940,0.04366) \\
\boldsymbol{b}^{*}=(0.11382,-0.14139,-0.22379) \\
\boldsymbol{c}^{*}=(-0.08131,0.21278,-0.17584)
\end{array}\right.
$$

(5) By measuring the reference lattice parameter of the $\alpha$ phase with high-energy synchrotron diffraction from 11-ID-C in APS, ANL, the reference state was known as $d_{0}=0.28805 \mathrm{~nm}$. With Eq. (1), the full strain tensor was then:

$$
\varepsilon=\left(\begin{array}{ccc}
-0.00172 & -0.00087 & 0.00028 \\
-0.00087 & 0.00033 & -0.00004 \\
0.00028 & -0.00004 & -0.00100
\end{array}\right)
$$

(6) The elastic modulus components of $\alpha$ phase are known as $C_{1}=236.9, C_{2}=140.6, C_{4}=116$. According to Hooke's Law, the stress-strain relationship is expressed as:

$$
\left\{\begin{array}{c}
\sigma_{33}=C_{2}\left(\varepsilon_{11}-\varepsilon_{\mathrm{c}}\right)+C_{2}\left(\varepsilon_{22}-\varepsilon_{\mathrm{c}}\right)+C_{1}\left(\varepsilon_{33}-\varepsilon_{\mathrm{c}}\right) \\
\sigma_{12}=C_{4} \varepsilon_{12} \\
\sigma_{23}=C_{4} \varepsilon_{23} \\
\sigma_{13}=C_{4} \varepsilon_{13}
\end{array}\right.
$$

where $\varepsilon_{c}$ representing the composition induced strain, $\sigma_{i j}$ and $\varepsilon_{i j}(i, j=1,2$ and 3$)$ representing the residual stress and strain in the crystal coordinates, respectively. In crystal coordinates, the stress matrix is

$$
\left(\begin{array}{lll}
\sigma_{11} & \sigma_{12} & \sigma_{13} \\
\sigma_{21} & \sigma_{22} & \sigma_{23} \\
\sigma_{31} & \sigma_{32} & \sigma_{33}
\end{array}\right)
$$



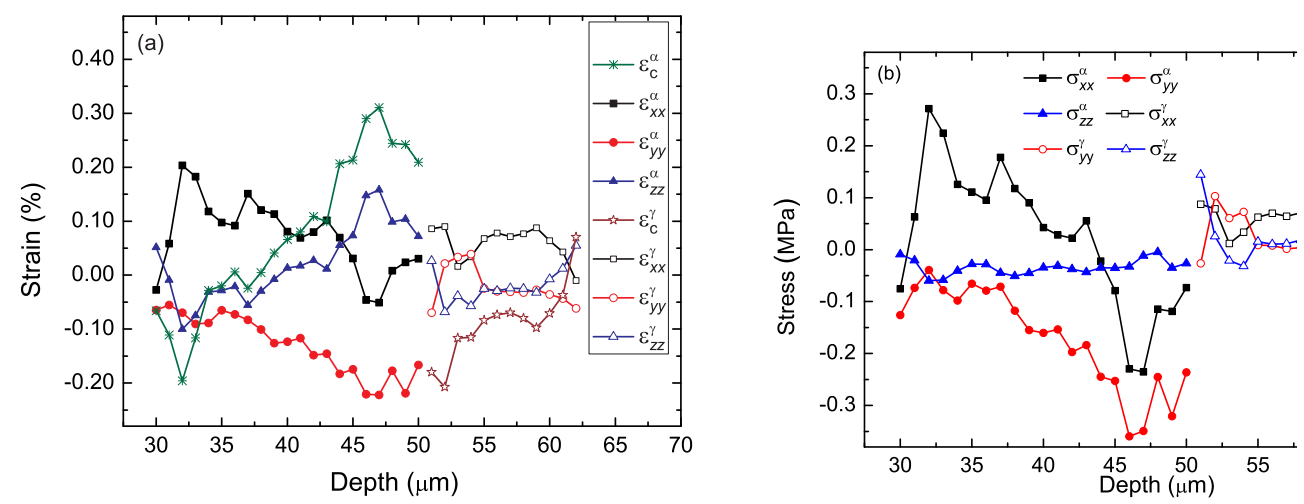

Fig. 4 Compositional and residual stress induced lattice strain evolutions along the depth (red line in Fig. 1) of the sample (a) and the accurate residual stress evolution along the sample depth (b)

When transforming to sample coordinates, calculation is described as:

$$
T_{\mathrm{cs}} \cdot \sigma_{i j} \cdot T_{\mathrm{cs}}^{-1}=\left(\begin{array}{ccc}
\sigma_{x x} & \sigma_{x y} & \sigma_{x z} \\
\sigma_{y x} & \sigma_{y y} & \sigma_{y z} \\
\sigma_{z x} & \sigma_{z y} & \sigma_{z z}
\end{array}\right)
$$

(7) Due to dog-bone sheet shape of the sample geometry, the sample's thickness is way thinner than the other two dimensions. Therefore, the stress condition in the sample can be simplified as plane-stress condition, namely . Combining Eqs.(3), (10) and (12), the compositional effect can be calculated as $\varepsilon_{\mathrm{c}}=-0.000451$. The residual stress induced strain $\varepsilon_{\mathrm{r}}$ is then known as

$$
\varepsilon_{\mathrm{r}}=\left(\begin{array}{ccc}
-0.001269 & -0.00087 & 0.00028 \\
-0.00087 & 0.000781 & -0.00004 \\
0.00028 & -0.00004 & -0.00549
\end{array}\right)
$$

Repeating procedure (1) to (7), $\varepsilon_{\mathrm{c}}$ and $\varepsilon_{\mathrm{r}}$ can be calculated at every position of the grain and in different depth.

The evolution of $\varepsilon_{\mathrm{c}}$ and $\varepsilon_{\mathrm{r}}$ from $\gamma$ phase to $\alpha$ phase (along the red line in Fig. 1) was plotted in Fig. 4(a). The green stars and the dark red stars represent the evolution of $\varepsilon_{\mathrm{c}}$ across $\alpha$ and $\gamma$ phase, respectively; while the other data represents the evolution of normal strain components induced by residual stress. And the calculated normal stress components of residual stress are also shown in Fig. 4(b). All stress/strain components are shown in sample coordinates for the purpose of a direct comparison with the applied tensile stress. It is clearly shown that the compositional effect results in a larger strain gradient than that of residual stress effect in both phases.

\section{Conclusions}

The present work quantitatively evaluated the lattice strain evolution inside individual grains of depth direction of two phases in a DPSS sample with the method of DAXM. The accurate lattice strain tensor, including the dilatation term and the deviatoric term can be calculated with pseudo-white-beam energy scan and plane stress condition in the sample. The main conclusions are as follows:

(1) The diffraction measured lattice strain is resulted from both the residual stress and composition variations. The contribution of residual stress will cause deviatoric lattice strain, while the effect of composition will lead to a dilatational lattice strain.

(2) DAXM method is able to quantify the contribution of compositional effect and the residual stress effect on the lattice strain measurement.

(3) In DPSS, the compositional effect weights more than that of residual stress on the measured lattice strain. The true residual stress should be evaluated with the removal of compositional effect.

\section{Acknowledgements}

This work was financial supported by the National Science Foundation of China (No. 51231002). The authors would like acknowledge that the Advanced Photon Source was supported by the U.S. Department of Energy, Office of Science, Office of Basic Energy Sciences, under Contract No. DE-AC02-06CH11357.

\section{REFERENCES}

[1] P.J. Withers and H. Bhadeshia, Mater. Sci. Technol. 17 (2001) 355.

[2] B. Clausen, T. Lorentzen and T. Leffers, Acta. Mater. 46 (1998) 3087.

[3] J. Matejicek, S. Sampath, P.C. Brand and H.J. Prask, Acta. Mater. 47 (1999) 607.

[4] M.R. Daymond and H.G. Priesmeyer, Acta. Mater. 50 (2002) 1613.

[5] L. Zwell, D.E. Carnahan and G.R. Speich, Metall. Trans. 1 (1970) 1007.

[6] F.H. Cocks and Ws. Radzinsk, Mater. Sci. Eng. A 7 
(1997) 302.

[7] M.S. Blanter, Phys. Status Solidi B 181 (1994) 377.

[8] X. Liu, F. Zhong and J.X. Zhang, Phys. Rev. B 52 (1995) 9970.

[9] S.J. Lee and Y.K. Lee, Scr. Mater. 52 (2005) 4.

[10] S.S. Babu, E.D. Specht, S.A David, J. Karapetrova, P. Zschak, M. Peet and H.K.D.H. Bhadeshia, Metall. Mater. Trans. A 36 (2005) 3281.

[11] H.G. Fu, Y.H. Qu and J.D. Xing, J. Mater. Eng. Perform. 18 (2009) 333.

[12] W.J. Liu, G.E. Ice, B.C. Larson, W.G. Yang, J.Z. Tischler and J.D. Budai, Metall. Mater. Trans. A 35
(2004) 1963.

[13] L.E. Levine, B.C. Larson, W.G. Yang, M.E. Kassner, J.Z. Tischler, M.A. Delos-Reyes, R.J. Fields and W.J. Liu, Nat. Mater. 5 (2006) 619.

[14] J.D. Budai, W. Liu, J.Z. Tischler, Z.W. Pan, D.P. Norton, B.C. Larson, W. Yang and G.E. Ice, Thin Solid Films. 516 (2008) 8013.

[15] Y.D. Wang, H.B. Tian, A.D. Stoica, X.L. Wang, P.K. Liaw and J.W. Richardson, Nat. Mater. 2 (2003) 101.

[16] G.E. Ice, J.S. Chung, W. Lowe, E. Williams and J. Edelman, Rev. Sci. Instrum. 71 (2000) 2001. 